\title{
Lung Magnetic Resonance Imaging with Diffusion Weighted Imaging Provides Regional Structural as well as Functional Information Without Radiation Exposure in Primary Antibody Deficiencies
}

\author{
Cinzia Milito $^{1}$ - Federica Pulvirenti ${ }^{1}$ - Goffredo Serra ${ }^{2}$ - Michele Valente ${ }^{2}$. \\ Anna Maria Pesce ${ }^{1} \cdot$ Guido Granata $^{1} \cdot$ Carlo Catalano $^{2} \cdot$ Francesco Fraioli $^{3,4}$. \\ Isabella Quinti ${ }^{1}$
}

Received: 18 February 2015 / Accepted: 25 May 2015 /Published online: 12 June 2015

(C) The Author(s) 2015. This article is published with open access at Springerlink.com

\begin{abstract}
Purpose Primary antibody deficiency patients suffer from infectious and non-infectious pulmonary complications leading over time to chronic lung disease. The complexity of this pulmonary involvement poses significant challenge in differential diagnosis in patients with long life disease and increased radio sensitivity. We planned to verify the utility of chest Magnetic Resolution Imaging with Diffusion-Weighted Imaging as a radiation free technique.

Methods Prospective evaluation of 18 patients with Common Variable Immunodeficiency and X-linked Agammaglobulinemia. On the same day, patients underwent Magnetic Resonance Imaging with Diffusion Weighted Imaging sequences, High Resolution Computerized Tomography and Pulmonary Function Tests, including diffusing capacity factor for
\end{abstract}

Cinzia Milito, Federica Pulvirenti and Goffredo Serra contributed equally to this work.

Electronic supplementary material The online version of this article (doi:10.1007/s10875-015-0172-2) contains supplementary material, which is available to authorized users.

Isabella Quinti

isabella.quinti@uniroma1.it

1 Department of Molecular Medicine, Sapienza University of Rome, Viale dell'Università 37, Rome, Italy

2 Department of Radiological, Oncological and Pathological Sciences, Sapienza University of Rome, Rome, Italy

3 Department of Nuclear Medicine, University College London Hospital, London, UK

4 Division of Imaging, University College London Hospital, London, UK carbon monoxide. Images were scored using a modified version of the Bhalla scoring system.

Results Magnetic Resonance Imaging was non-inferior to High Resolution Computerized Tomography in the capacity to identify bronchial and parenchymal abnormalities. HRCT had a higher capacity to identify peripheral airways abnormalities, defined as an involvement of bronchial generation up to the fifth and distal (scores 2-3). Bronchial scores negatively related to pulmonary function tests. One third of consolidations and nodules had Diffusion Weighted Imaging restrictions associated with systemic granulomatous disease and systemic lymphadenopathy. Lung Magnetic Resolution Imaging detected an improvement of bronchial and parenchymal abnormalities, in recently diagnosed patients soon after starting Ig replacement.

Conclusions Magnetic Resonance Imaging with Diffusion Weighted Imaging was a reliable technique to detect lung alterations in patients with Primary Antibody Deficiencies.

Keywords Primary antibody deficiencies $\cdot$ lung abnormalities $\cdot$ magnetic resonance $\cdot$ diffusion weighted imaging $\cdot$ inflammation $\cdot$ GLILD

\section{Introduction}

Patients with X-linked Agammaglobulinemia (XLA) and Common Variable Immunodeficiency (CVID) suffer from recurrent respiratory infections and non-infectious pulmonary diseases possibly leading, over time, to permanent lung damage [1-4]. Common lung alterations are bronchiectasis, bronchial wall thickening, nodules and parenchymal consolidations [5, 6]. Moreover, patients with primary antibody deficiencies (PAD) are affected also by a group of lung diseases, 
denominated granulomatous-lymphocytic interstitial lung disease (GLILD) [7-11] characterized by granulomatous and pulmonary lymphoid hyperplasia, histological patterns consisting of lymphocytic interstitial pneumonia (LIP), follicular bronchiolitis, and lymphoid hyperplasia [12]. The complexity of this pulmonary involvement poses a significant challenge in differential diagnosis [13]. Physical examination, laboratory analysis and Pulmonary Function Tests (PFTs) are routinely used in the work-up of PAD lung disease, but High Resolution Computed Tomography (HRCT) is often required to establish a definite diagnosis. HRCT scan is the gold standard for the assessment of lung diseases. Because of the long life course of PAD, patients undergo a considerable number of radiological examinations during their life. As an increased radio sensitivity has been described [14-19], clinicians should consider a risk-benefit assessment when ordering a CT scan. We have already shown that Magnetic Resonance Imaging (MRI), a radiation-free technique was complementary or alternative to HRCT scan [20] to detect lung alterations. However, chest HRCT scan and morphological MRI sequences did not give information on the presence of foci of active inflammation and often invasive methods as lung biopsy or bronchoalveolar lavage are necessary [13]. Functional MRI with diffusion-weighted imaging (DWI) sequences might represent a promising tool for the identification of areas of active inflammation/sub clinical infections in lung parenchyma [21]. DWI signal derives from the free motion of water molecules in tissues. The interaction with an increased number of cells or macromolecules leads to a restriction of this motion, with higher DWI signal intensity. DWI has been validated as diagnostic tool in various inflammatory diseases in the abdomen, pelvis and brain [22-24].

In lung imaging, DWI has been applied in the differential diagnosis of lung consolidations and neoplasms, based on the fact that the signal intensity is higher in viable tumour tissues than in less densely packed tissues, such as tumour necrosis or benign consolidations [25-27].

Following these considerations, we have introduced DWI in our diagnostic approach for PAD patients. The objective of this study was to evaluate if chest MRI with DWI may represent a feasible radiation free option in the evaluation of PAD lung disease.

\section{Methods}

Study Design We set out a prospective single center study, enrolling 18 consecutive patients aged $>18$ years with an established diagnosis of PAD [28] and recurrent respiratory infection. On the same day, patients underwent chest MRI without contrast medium and including DWI sequences and Pulmonary Function Tests (PFTs), including diffusing capacity factor for carbon monoxide $\left(\mathrm{D}_{\mathrm{LCO}}\right)$. To validate the MRI score, a chest HRCT scan was performed. Examinations were carried out far from clinical evidence of acute pulmonary infection. Data on clinical manifestation and immunoglobulin replacement were collected by medical records. Immuno-phenotyping of peripheral blood mononuclear cells was also performed. The institutional review board approved the study and a signed informed consent was obtained from all participants.

HRCT Study HRCT images were acquired with a 64-detector row spiral CT scanner (Volume Sensation Cardiac; Siemens), without injection of IV contrast agent, from the lung apices to the upper abdomen in a single breath-hold at the end of full inspiration. The parameters used for acquisition were as follows: $100 \mathrm{kV}$, CAREdose (Siemens) with quality reference set at $100 \mathrm{mAs}$; collimation, $64 \times 0.6 \mathrm{~mm}$; gantry rotation time, $0.33 \mathrm{~s}$; scan time, $6 \mathrm{~s}$; reconstruction kernel, B30 (for mediastinal evaluation) and B60 (for lung parenchyma evaluation).

MRI Study MRI images without contrast medium were obtained with a 1.5 Tesla scanner (Magnetom Avanto, Siemens, Enlargen, Germany) using a respiratory triggered DP weighted BLADE sequence (TR:2000 ms; TE $=27 \mathrm{~ms}$; FOV $400 \mathrm{~mm}$; flip angle: $150^{\circ}$; slice thickness $5 \mathrm{~mm}$ ) acquired on an axial plane. DWI sequence (TR: $5632 \mathrm{~ms}, 12$; TE: $83 \mathrm{~ms}$; flip angle: $90^{\circ}$; FOV $400 \mathrm{~mm}$ slice thickness $5 \mathrm{~mm}$; b0-b800 $\mathrm{s} / \mathrm{mm} 2$ ) on an axial plane was included in the acquisition protocol. A combination of two surface coils covering the whole thorax and the spine was used.

Image Analysis MRI and CT scan studies were scored adopting the Bhalla score $[20,29]$ in a random order by two chest radiologists, blinded to patients' identities and clinical information. Readers evaluated each examination independently and separately and reached an agreement for the controversial lesions. In order to reduce recall bias effects from the remembering of MRI findings, HRCT scans were evaluated 4 weeks after the analysis of the MR images. DWI abnormalities (hot spots) were identified as areas showing equal or higher signal intensity compared to the spinal cord at the highest $b$ value [26]. An eyeballing comparison was performed between DWI and morphological-MRI in order to identify the morphological substrate of the diffusion abnormalities observed in consolidations or nodules.

Bhalla Score Classical Bhalla score evaluated severity and extent of bronchial and parenchymal parameters [29]. Bronchial parameters were: a) bronchiectasis severity, b) extent of bronchiectasis (number of lung segments), c) peribronchial thickening, d) extent of mucus plugs (number of lung segments), e) generation of bronchial division involved (bronchiectasis/plug). Parenchymal parameters were: a) abscesses or sacculations (number of lung segments), b) numbers of bullae, c) emphysema (number of lung segments), d) collapse/consolidations. Two extra parenchymal categories were introduced 
for the assessment of nodules: nodule number (score 0: no nodules detected; score 1: $<5$ nodules detected; score 2: $>5$ nodules detected) and size: (score 1: $<1 \mathrm{~cm}$; score 2: $>1 \mathrm{~cm}$ ). The cut-off diameter discriminating between nodules and consolidations was up to $30 \mathrm{~mm}$. Total bronchial score ( $\min 0$; $\max$ 15) was defined as the sum of each bronchial parameter score; total parenchymal score (min 0; $\max 14)$ was defined as the sum of each parenchymal parameter score; total Bhalla score was defined as the sum of bronchial and parenchymal scores.

PFTs The PFTs were performed at the Pulmonary Function Unit. We analysed the following data sets: 1) forced vital capacity (FVC), 2) forced expiratory volume in one second (FEV1); 3) forced expiratory flow between 25 and $75 \%$ (FEF25-75); 4) corrected $\mathrm{D}_{\text {LCO. }}$.

Flow Cytometry Analysis Peripheral blood mononuclear cells were obtained by density-gradient centrifugation.
Immunophenotyping was performed with the combination of 4 fluorochrome-labeled monoclonal antibodies (BD Biosciences). The following B-cell populations were analysed: classical naïve $\left(\mathrm{CD} 19^{+} \mathrm{CD} 27^{-} \mathrm{CD} 21^{+} \mathrm{CD} 38^{+}\right)$, switched memory $\left(\mathrm{CD} 19^{+} \mathrm{CD} 27^{+} \mathrm{CD}^{+} 1^{+} \mathrm{IgM}^{-}\right)$, IgM memory $\left(\mathrm{CD} 19^{+} \mathrm{CD} 27^{+} \mathrm{Ig} \mathrm{M}^{+} \mathrm{Ig} \mathrm{D}^{+}\right)$, transitional $\left(\mathrm{CD} 19^{+} \operatorname{IgM}^{++} \mathrm{CD} 38^{++}\right)$and $\mathrm{CD} 21^{\text {low }}\left(\mathrm{CD} 19^{+} \mathrm{CD} 21^{-1}\right.$ $\left.{ }^{\text {low }} \mathrm{CD} 38^{-}\right)$. Dead cells were excluded from analysis by side/ forward scatter gating. FACS analyses were performed on a FACSCalibur instrument (BD Biosciences) using the CellQuest (BD) and FlowJo (Tree Star) software.

Statistical Analysis Statistical analysis was performed by using a dedicated software (StatView). Descriptive data were presented as median and interquartile range (IQR), as indicated. Comparison of clinical and radiological changes between groups, was performed by Mann-Whitney test. Comparison of categorical and non-continuous variables between groups was performed
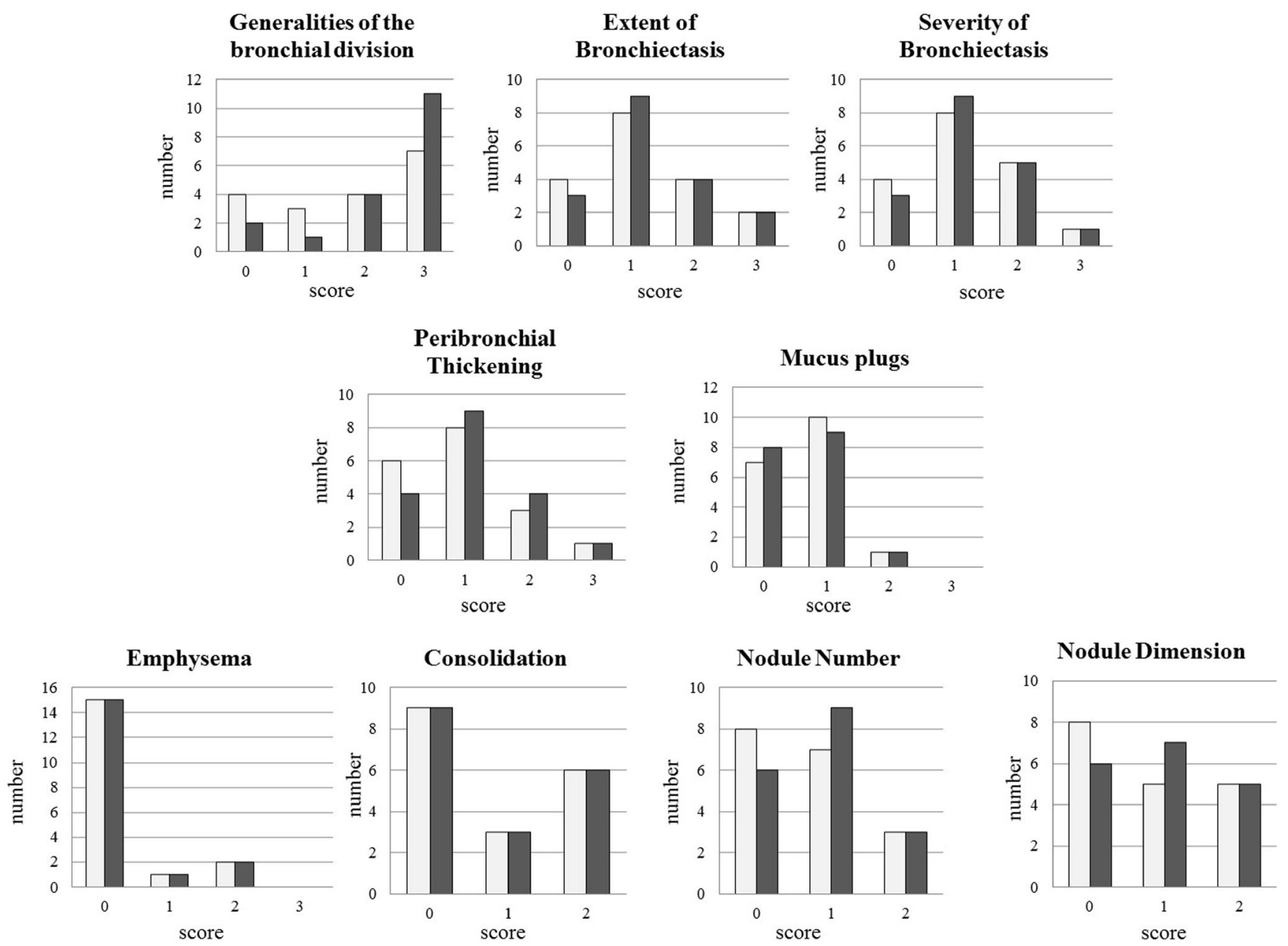

Fig. 1 HRCT and MRI score comparison a Number of patients with primary antibody deficiency and defined bronchial abnormality score (generalities of the bronchial division, bronchiectasis severity, extent of bronchiectasis, peribronchial thickening, mucus plugs) identified by HRCT scan and MRI. b Number of patients with primary antibody

deficiency and defined parenchymal abnormality score (abscesses, consolidations, bullae, emphysema, nodules number and dimension) identified by HRCT scan and MRI. Gray bars refer to MRI data; black bars refer to HRCT scan data 
by chi-square test. Comparison of MRI and HRTC scores was performed by chi square test to assess MRI non-inferiority. Comparison between single PFTs parameter and MRI score was assessed by simple linear regression analysis. A p value of $<0.05$ was taken as the threshold of statistical significance.

Inter-observer agreement was evaluated comparing the HRCT and MRI total scores (TOT) calculated by the 2 readers; intra-observer variability was evaluated comparing the two different readings of the same HRCT and MRI datasets performed by the most experienced radiologist after an interval of at least 4 weeks. The intra- and inter-observer agreement for scoring values were analysed by the use of Intraclass Correlation Coefficient (ICC).

\section{Results}

Patient Population Eighteen (13 males, 5 females) PAD patients were enrolled in the study: 16 patients had a diagnosis of CVID and two patients of XLA. The median age at the study time was 39.5 years (IQR 30.5-55). At the enrolment, 14 patients received Ig replacement therapy administered intravenously (IVIG) and two patients subcutaneously (SCIG); 2 patients (n.4 and 9) were newly diagnosed CVID and they started the IVIG substitutive treatment at the time of the study, reaching a plateau of trough $\operatorname{IgG}$ level 3 months thereafter. PAD-associated clinical conditions were: autoimmune disease (8/18 patients), chronic lymphadenopathy (10/18 patients), splenomegaly (11/18 patients), systemic granulomatous disease (seen by histology - 4/18) and chronic obstructive lung disease (COPD) (7/18 patients). Relevant clinical and immunologic data were summarized in Supplementary Table 1. All patients with COPD diagnosis were on treatment with longacting inhaled therapy (beta-agonists and steroids and/or anticholinergics). None of our patients were on treatment with systemic steroids and/or immunosuppressive drugs. Overall, on PFTs, FEV1 was $68 \%$ (IQR 45-85\%), FVC: $70.5 \%$ (IQR 53-81 \%), FEV1/FVC: 97 \% (IQR 73-107 \%), PEF: $64 \%$ (IQR 43-79 \%), FEF 25-75: $71 \%$ (IQR 32-98 \%); DLCO (corrected): $91.6 \%$ (IQR 78.6-96.7\%).

CT and MRI: Image Acquisitions Room time for CT scan was $4.1 \mathrm{~min}$ (IQR 3.9-5.2) and the dose length product was $104 \mathrm{mGy} \times \mathrm{cm}$ (IQR: 90-113); effective dose was $1.3 \mathrm{mSv}$ (IQR: 0.9-1.8). MRI room time was $17 \mathrm{~min}$ (IQR 12-20). Intra-observer agreement was calculated to assess reproducibility of the newly developed DWI scoring system. A good interobserver agreement was found for both bronchial and parenchymal MRI scores (ICC for Total Score: 0903 and 0942 respectively). A good inter-observer agreement was observed for DWI scores (ICC for Total DWI score: 0975). A good inter-observer agreement between the two readers was found in the scoring evaluation of HRCT studies (ICC for Total Score: 0971).
Lung Abnormalities by MRI Mean total score recorded by MRI was 8 (IQR 5-11). Bronchial abnormalities and parenchymal abnormalities were evident in $78 \%$ and in $66.7 \%$ patients, respectively. Individual bronchial and parenchymal MRI scores were shown in Supplementary Table 2. Mean total bronchial MRI score was 6 (IQR 3.25-9). Bronchiectasis, mucus plugging, peribronchial thickening were detected in $77.8,55.5$ and $66.7 \%$ of patients, respectively. As expected, PAD patients with COPD had higher total MRI bronchial scores (9, IQR 7-11) in comparison to patients without COPD (5, IQR 1.5-6) $(p=0.033)$. Total MRI parenchymal scores were 2 (IQR $0-6$ ). None of the patients had bullae or abscesses; $16.7 \%$ patients had emphysema. Consolidations and nodules were recorded in 50 and $55.5 \%$ patients, respectively. The cumulative number of consolidations was 41 and of nodules was 40 .

\section{HRCT and MRI Comparison in Lung Abnormalities Evaluation}

Bronchial abnormalities were the most common lung alterations recorded in PAD patients by HRCT scan ( $89 \%$ ). We observed high levels of concordance between HRCT and MRI in scoring bronchiectasis severity $(p=0.0004)$, extent of bronchiectasis $(p<0.0001)$ and mucus plugging $(p<0.0001)$. A lower but still significant level of concordance was found between HRCT and MRI in the capacity to score the bronchial division involvement $(p=0.02)$, in that HRCT had a higher capacity $(p=0.02)$ to identify peripheral airways abnormalities, defined as an involvement of bronchial generation up to the fifth and distal (scores 2-3) detected in 83 and $61 \%$ of patients, respectively.

We observed high levels of concordance between HRCT and MRI in scoring parenchymal abnormalities: consolidation, $p<0.0001$; nodule number, $p<0.0001$; nodule dimension, $p<0.0001$; emphysema, $p<0.0001$ ). Ten out of 18 patients had at least a nodule identified by HRCT and MRI. Nodule dimension was similarly scored with exception of two patients with small nodules (diameter $<1 \mathrm{~cm}$ ) identified by HRCT scan only. Figure 1 shows the comparison between HRCT scan and MRI scores. Supplementary Table 2 shows individual scores by MRI and HRCT scan.

PFTs and Lung Radiological Abnormalities by MRI Overall, a statistically significant negative correlation was found between total bronchial scores and PFTs parameters (FEV1, $r 2=0.42 p=0.003$; FVC, $r 2=0.45 p=0.002$; PEF, $r 2=0.4 p=0.006$ ). In detail, FEV1 was negatively related with all bronchial parameters; FVC was negatively related with

Fig. 2 Pulmonary Function Test and Bhalla bronchial score Linear regression analysis of PFTs and bronchial parameters by MRI scored according to Bhalla; $r 2$ and $\mathrm{p}$ value are indicated for each parameter 

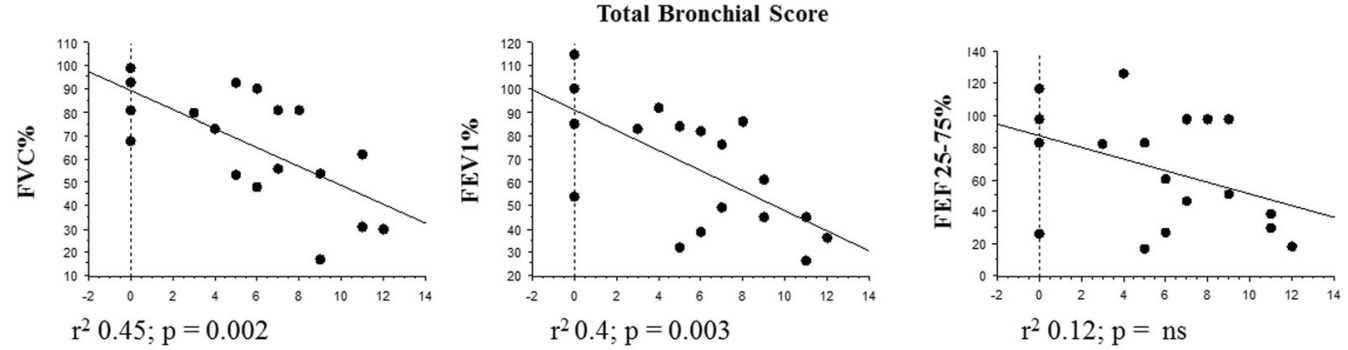

Generalities of Bronchial Involvement
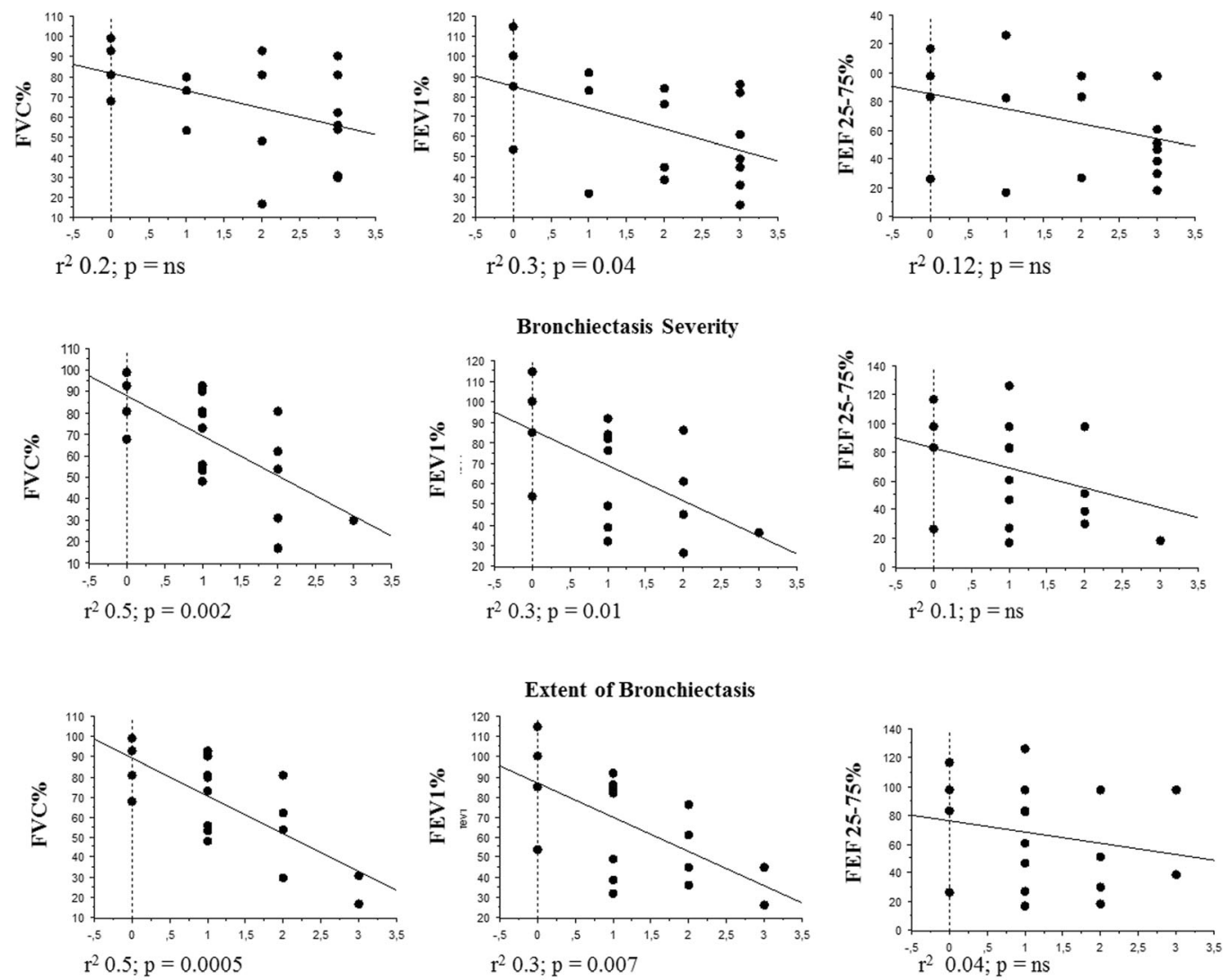

Peribronchial Wall Tickening
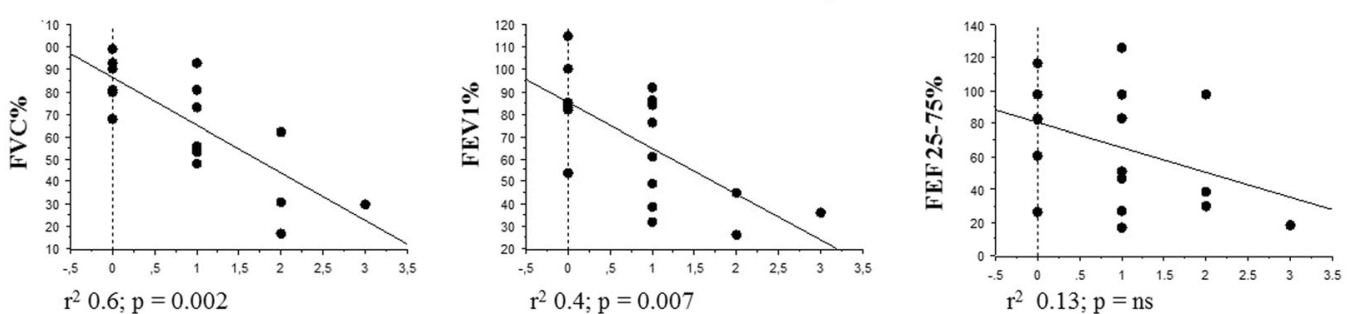

Mucus Plugging
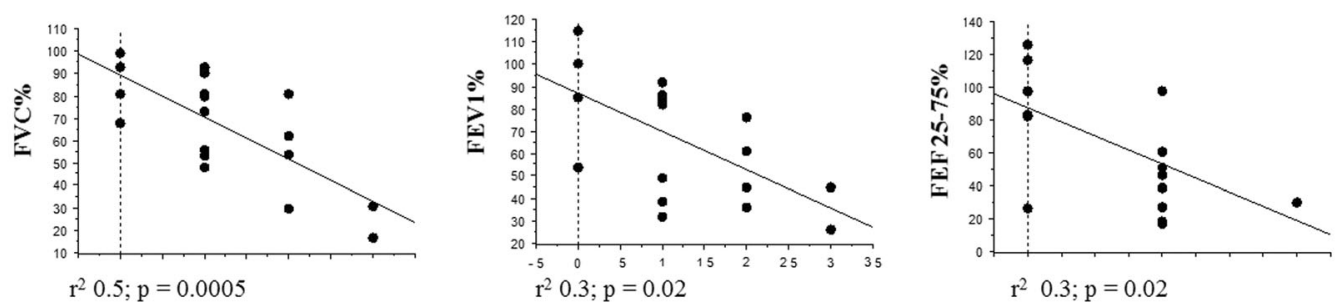


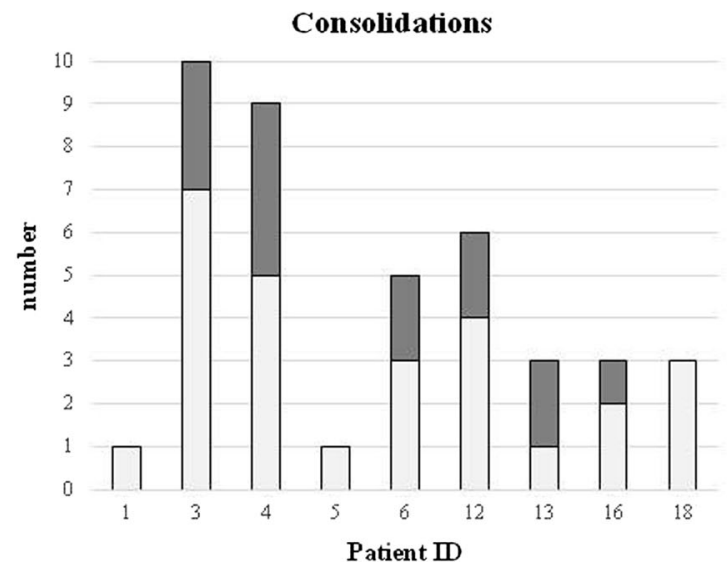

Fig. 3 Parenchymal abnormalities with and without DWI hotspots Number of consolidations (a) and nodules (b) with or without DWI hot spots in PAD patients. Grey box refer to the number of lung abnormalities

bronchiectasis severity, bronchiectasis extension, bronchial wall thickening; FEF 25-75 was negatively related with mucus plugging only (Fig. 2). Three patients (n. 4, 5, 10) with normal PFTs parameters had bronchial abnormalities (mucus plugging and bronchial wall thickening) detectable by MRI.

Total parenchymal scores did not show any correlation with PFTs, including $\mathrm{D}_{\mathrm{LCO}}$.

MRI DWI Study Six out of nine patients with consolidations recorded by MRI had DWI hotspots. Overall, 14 out of 41 consolidations recorded $(34.1 \%)$ were DWI restricted (Fig. 3a). DWI hotspots were related to consolidations with a

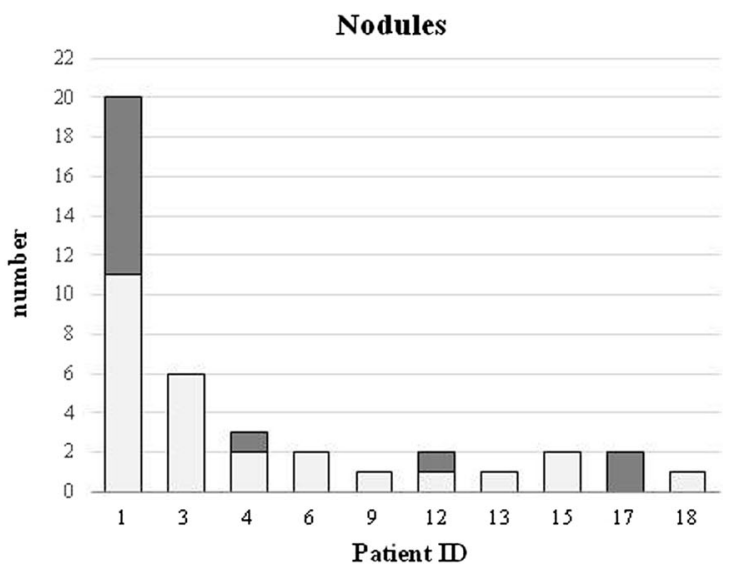

without DWI hotspots; black box refer to the number of lung alterations with DWI hotspots (y-axis). Numbers on the x-axis refer to patients identification number (ID)

MRI score $<1$ (segmental or lobar extension). All consolidations with a MRI score of 1 (sub-segmental extension) were DWI negative. Four out of ten patients with nodules had DWI hotspots. Overall, 13 out of 40 nodules recorded by MRI (32.5 \%) were DWI-restricted (Fig. 3b). Nodules had a maximum transversal diameter ranging between 3 and $14 \mathrm{~mm}$. There was no difference in the mean diameter between nodules with or without DWI hotspots (7 mm, IQR 6-8, vs $5.5 \mathrm{~mm}$, IQR 4.75-7). In Fig. 4 and Fig. 5, we show representative MRIs with DWI-restricted and DWI-non restricted areas. Patients with DWI hotspots had higher total MRI Bhalla score and higher total MRI parenchymal scores in comparison to
Fig. 4 Representative MRI with DWI sequences: consolidations Consolidations detected by MRI with DWI. MRI BLADE sequence showed bilateral, chronic collapses of lower lobes (white arrow) (a) without DWI corresponding hotspots (b). MRI BLADE sequence showed a consolidation (white arrow) of the left lower lobe (c) with a corresponding hotspot at the DWI assessment (white arrow) (d). The histology of this consolidation by lung biopsy is shown in Supplementary Fig. 1
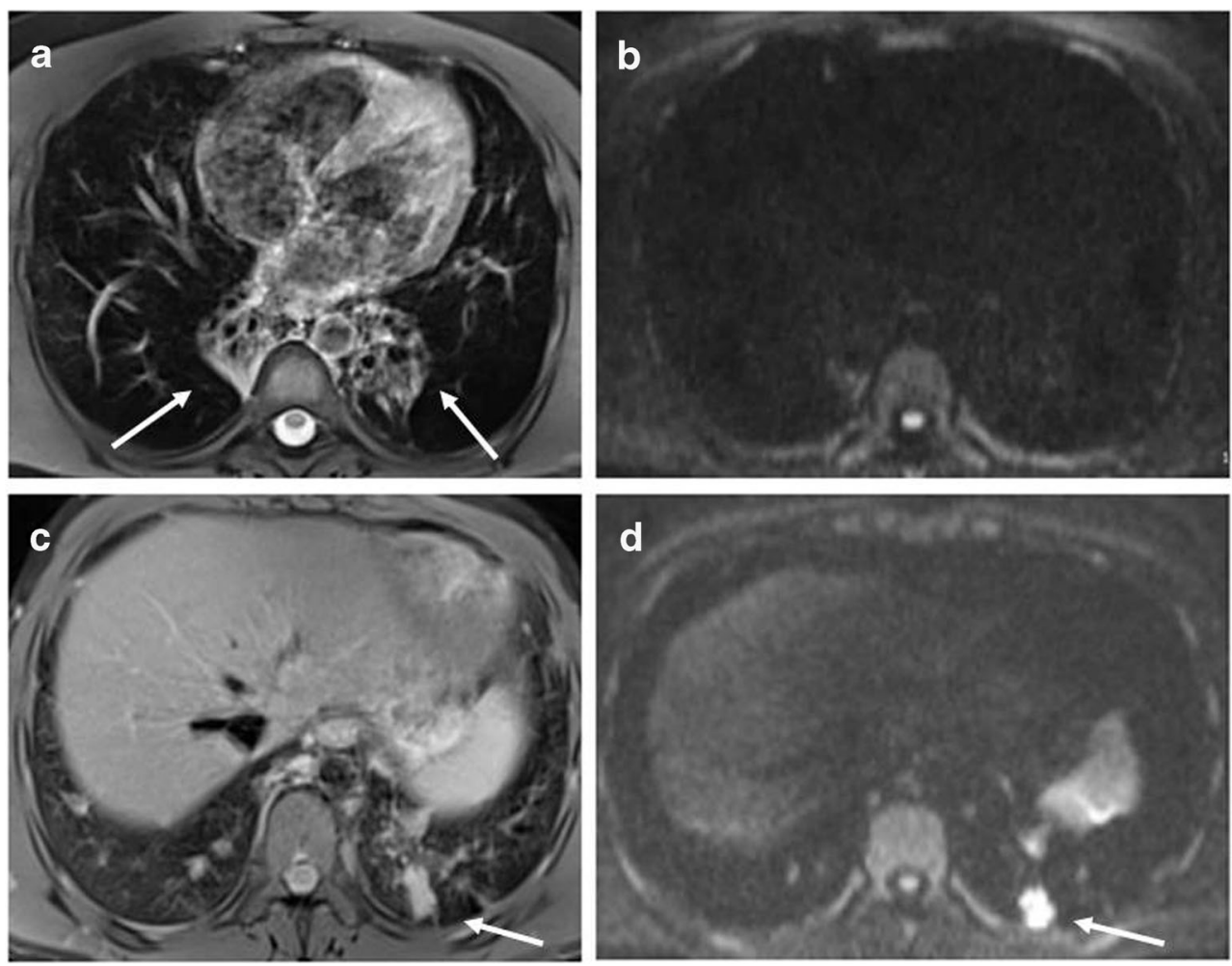
Fig. 5 Representative MRI with DWI sequences: nodules Nodules detected by MRI with DWI. MRI BLADE sequence detecting a nodule (white arrow) at the right lower lobe (a) without a DWI corresponding hotspot (b). MRI BLADE sequence detecting multiple and bilateral small nodules of the lower lobes (c) and their corresponding hotspots at DWI assessment (white arrow) (d)
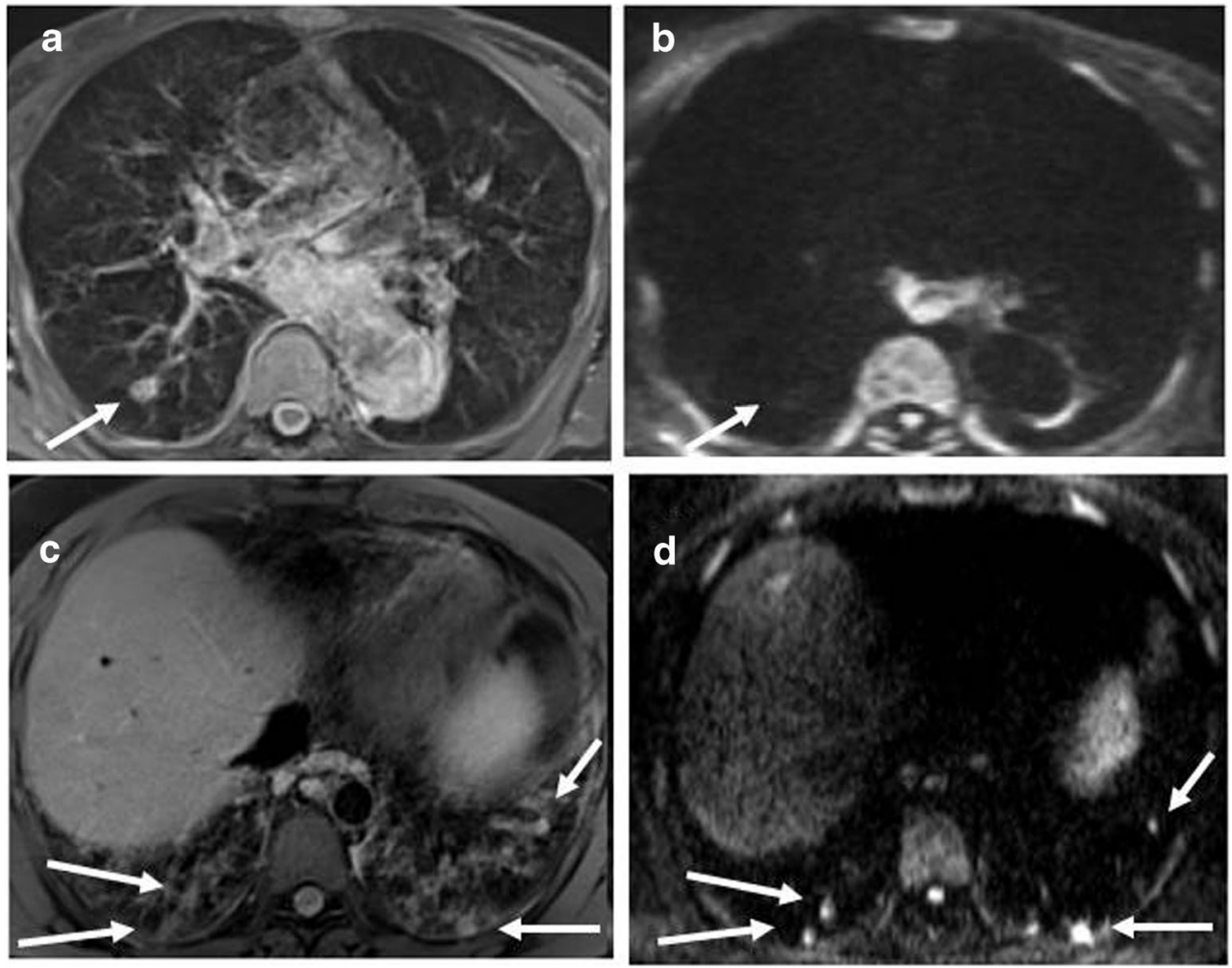

patients who did not have DWI-restricted parenchymal areas (total score 10 (IQR 7.5-16) vs 6 (IQR 2.5-8.5) ( $p=0.05)$; total parenchymal score 5 (IQR 3.5-6) vs 0 (IQR 0-0.75), respectively). Patients with DWI-restricted consolidations had also a lower predicted FEV1\% in comparison to patients without DWI hotspots (45\%, IQR 34-71\% vs $81 \%$, IQR $60.8-$ $85 \%$ ). DWI restricted abnormalities (nodules and/or consolidations) were only detected in patients with systemic granulomatous diseases. Isolated DWI restricted consolidations were mainly present in patients with systemic lymphadenopathy ( $85 \%$ vs $36 \%, p=0.04)$. The presence of DWI-restricted areas was not associated with splenomegaly and autoimmune manifestations. Moreover, patients with DWI-restricted abnormalities had an increased percentage of CD2 $1^{\text {low }} \mathrm{B}$ cells in comparison to patients without DWI hot spots (33\%, IQR 23.5-47, vs $16 \%$, IQR 14-21, $p=0.05$ ). The analysis of other B and T cell subsets did not show any differences between patients with DWI restricted and not restricted areas.

Only one patient (n. 4) underwent a lung biopsy of in a DWI-restricted consolidation for a suspected lung lymphoma. The histological pattern showed multifocal interstitial lymphoid infiltrates spreading into the alveolar septa and surrounding airways and vessels, consistent with LIP (Supplementary Fig. 1).

Newly Diagnosed Patients Patients (n. 4, 9), who started IVIG replacement therapy at the study time, had a second lung MRI assessment and PFTs 12 months after starting Ig treatment. FEV1 improved in both patients $(+8$ and $+7 \%$, respectively) as well as total parenchymal and total bronchial scores (Supplementary Fig. 2). Representative MRI sequences in a patient before and 12 months after Ig administration were shown in (Fig. 6).

\section{Discussion}

In this study, we demonstrated that MRI with DWI identified lung inflammatory lesions in patients with PAD. Moreover, we confirmed our previous data on the non-inferiority of MRI vs HRCT scan [29]. HRCT is the gold standard in the lung structural damage evaluation for its excellent spatial resolution, fast acquisition times and wide availability [30]. However, HRCT poses concerns regarding the radiation dose, mostly in CVID patients, a population with a suggested increased radio sensitivity undergoing a long life follow-up [14-19]. Therefore, for these subjects, radiation-free imaging would be desirable for both follow-up and therapy monitoring. Nowadays, several strategies have been developed to overcome the inherent technical limitations of lung MRI and this imaging modality is becoming a promising tool in different diseases, including primary immune deficiencies such as ataxia-telangiectasia [31]. The implementation of DWI sequences enabled the acquisition of valuable information regarding the microstructure of tissues, allowing the identification of several pathological processes, including active inflammation [21-24, 32, 33]. In a previous cross-sectional 
Fig. 6 Representative MRI sequences in a patient at diagnosis and after 12 months In a 18 years old man who started Ig replacement therapy (patient n. 4), MRI BLADE sequence at first MRI showed multiple distal mucus plugs with branching patterns ("tree in bud" appearance) of the left upper lobe (white arrows) (a); these abnormalities regressed at the second MRI assessment after 12 months (white arrows) (b). MRI BLADE sequence at first MRI showed one nodule (white arrow) at the right lower lobe (c); the nodule showed a reduction in size at the second MRI assessment after 12 months (white arrow) (d)
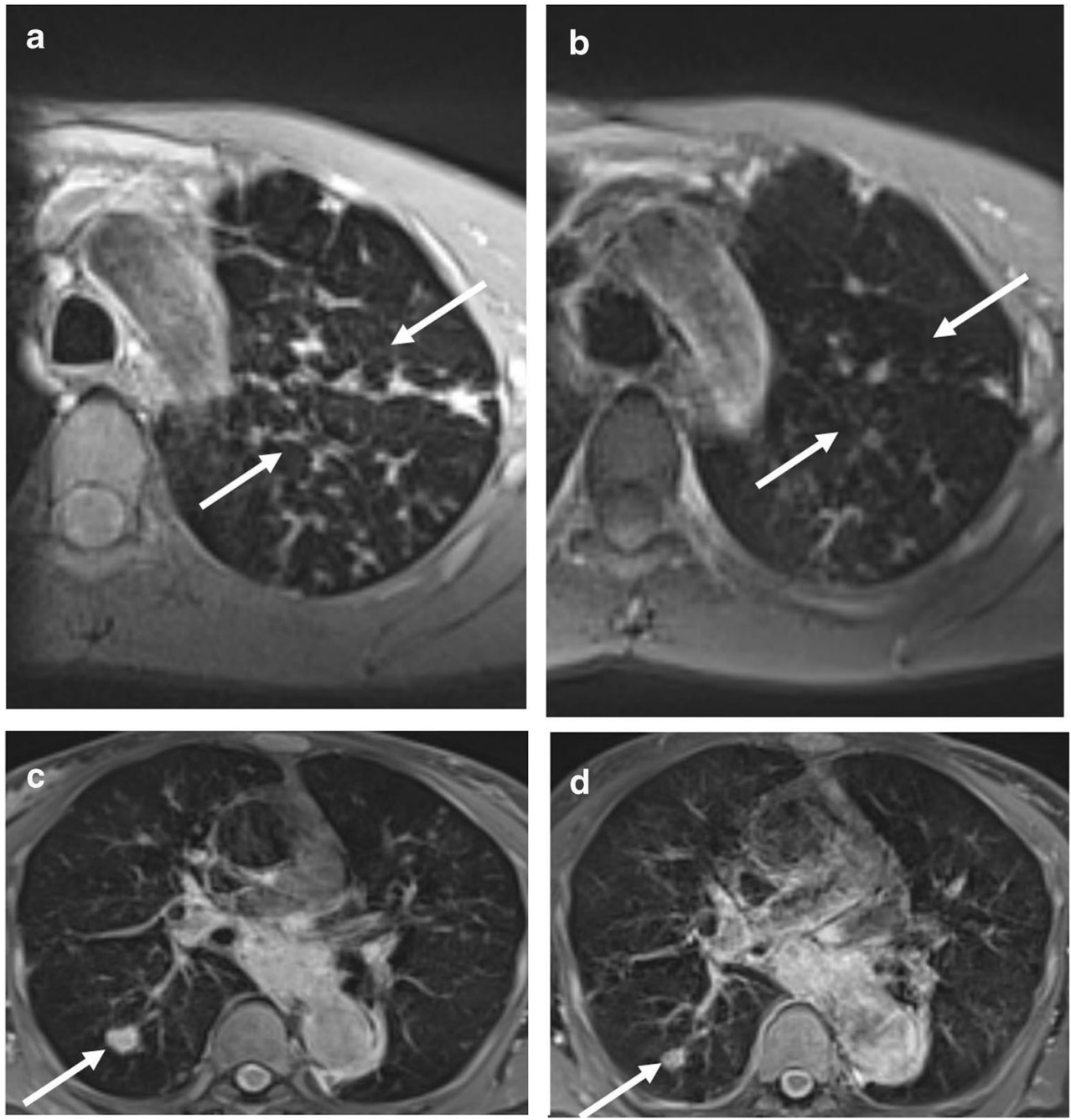

study, we have demonstrated that MRI with BLADE sequences was a reliable technique in the detection of bronchial and parenchymal alterations and provided information overlapping HRCT [20]. We confirmed our previous data and we showed high levels of concordance between HRCT and MRI in scoring bronchiectasis severity, extent of bronchiectasis and mucus plugging. However, HRCT had a higher capacity to identify the involvement of bronchial generation up to the fifth and distal. We showed here that the inclusion of DWI sequences might provide valuable records to detect the presence of areas of active inflammation. Other radiological procedures, such as Positron Emission Tomography-CT, have been proposed for localization and quantification of active inflammation in the lung [34, 35]. However, their use is restricted by high ionizing radiation exposure, cost and limited availability.

For DWI image analysis, we used an adapted Bhalla scoring system taking into account all the alterations showing equal or higher signal intensity compared to the spinal cord at the highest $b$ value. This technique, already adopted in previous experiences [36], did not imply a quantitative analysis derived from Apparent Diffusion Coefficient maps, it is hardly adaptable to lung imaging for its low proton density, B0 inhomogeneity, and physiologic motion. Moreover, the presence of susceptibility artefacts would lead to difficulty and poor reproducibility in selecting a consistent region of interest.

In this cohort, MRI protocol was able to identify lung alterations occurring in PAD patients; our results showed that a semiquantitative assessment with a scoring system was feasible and matched well with the total mean HRCT scores and PFTs of the same patients. In our cohort, the MRI evaluation showed that bronchial abnormalities were the prevalent finding in PAD patients. These abnormalities might be considered as signs of chronic, active inflammation and/or infections. [1-5]. Thus, as previously suggested [3] aside from Ig replacement, a strategy to reduce the chronic mucosal damage should be defined. Chronic antibiotics treatment was suggested but its efficacy is still matter of discussion [37]; now we are conducting a large double blind study about prophylaxis with azithromycin in PAD in order to prove a possible benefit in reducing respiratory exacerbations as shown in patients with non-cystic fibrosis bronchiectasis [38]. 
Even if Ig therapy could not reduce the progression of lung diseases and damage, we documented an improvement of parenchymal and bronchial scores and of PFTs in newly diagnosed PAD patients after the beginning of Ig replacement therapy. We found a significant relationship between the extent of MRI bronchial abnormalities score and PFTs. Based on these findings, it appears that PFTs alterations were strictly connected to a bronchial damage. However, MRI parenchymal scores did not correlate with PTFs, including $\mathrm{D}_{\mathrm{LCO}}$. This was not surprising since none of the patients enrolled had bullae, only few patients had a mild emphysema and none had clinical signs of acute pulmonary infection at the time of the study. Moreover, MRI was able to identify active bronchial abnormalities in a group of patients with normal PFTs. The interpretation of DWI findings may represent a useful tool in addition to the conventional morphological analysis, mostly in subjects with advanced disease. In our evaluation, all DWI hotspots corresponded to morphological alterations, but not vice versa: lung pathological findings showed different DWI patterns or no DWI signal at all. It can be postulated that the mismatch between DWI and morphological data correlates with cellular and extra-cellular space modifications (density/composition/water content) induced by active inflammation: these phenomena may affect DWI even before macroscopic alterations can be detected on conventional imaging. DWI parenchymal restricted areas were mainly detected in patients with severe total and parenchymal scores and with more severe alterations of PFTs. They were associated with abnormalities described in patients with the inflammatory CVID phenotypes: systemic granulomatous disease, systemic lymphadenopathy, expansion of $\mathrm{CD} 21^{\text {low }} \mathrm{B}$ cells [39]. For these reasons, DWI might represent a marker of disease activity and it could be used during followup of PAD patients in order to distinguish foci of active inflammation/sub-clinically progressive lung disease from chronic pathological manifestations [8-11]. The presence of inflammation represents an important pathological process underlying many lung morphological alterations in PAD patients and, in the clinical practice, it is important to determine the activity of disease to guide interventions. DWI sequences, with the possible identification of active inflammation, may allow an early discrimination of patients with infective/inflammatory or non-infective/inflammatory disease. Inflammatory lung diseases in CVID have been reported to be associated with lung disease progression and increased mortality [11, 40, 41], but the timing when starting an immunosuppressive treatment is still open [42-44]. Moreover, since MRI is a radiation-free technique, it can be repeatedly used in follow-up monitoring of treatment response, differentiating between active and fibrotic lesions.

\section{Conclusions}

Clinicians might consider MRI with DWI in the diagnostic algorithm of pulmonary lesions as a non-invasive, radiation- free method complementary to other diagnostic tools. Our results might help to define the role of morphological MRI sequences and DWI sequences in the diagnosis of foci of active inflammation and/or sub-clinically progressive lung disease. The major limit of this study was the lack of information on bronchoalveolar lavages and the presence of a single DWI restricted lung biopsy. Future studies could likely benefit from the longitudinal evaluation of larger cohorts of patients with primary immune deficiencies or other clinical conditions requiring a frequent ionizing radiation exposure in order to assess the potential use of DWI to select biopsy areas and to monitor the response to treatment in non-infective inflammatory disease.

Acknowledgments We acknowledge our patients, and the Jeffrey Modell Foundation. Funding: Progetto ricerche Universitarie, 2011, 2012, 2013 and Euro-PADnet-FP7-HEALTH-2007-A.

Open Access This article is distributed under the terms of the Creative Commons Attribution 4.0 International License (http://creativecommons.org/licenses/by/4.0/), which permits unrestricted use, distribution, and reproduction in any medium, provided you give appropriate credit to the original author(s) and the source, provide a link to the Creative Commons license, and indicate if changes were made.

\section{References}

1. Cunningham-Rundles $\mathrm{C}$, Bodian C. Common variable immunodeficiency: clinical and immunological features of 248 patients. Clin Immunol. 1999;92:34-48.

2. Quinti I, Soresina A, Spadaro G, Martino S, Donnanno S, Agostini $\mathrm{C}$, et al. Long-term follow-up and outcome of a large cohort of patients with common variable immunodeficiency. J Clin Immunol. 2007;27:308-16.

3. Quinti I, Soresina A, Guerra A, Rondelli R, Spadaro G, Agostini C, et al. Effectiveness of immunoglobulin replacement therapy on clinical outcome in patients with primary antibody deficiencies: results from a multicenter prospective cohort study. J Clin Immunol. 2011;31:315-22.

4. Costa-Carvalho BT, Wandalsen GF, Pulici G, Aranda CS, Solé D. Pulmonary complications in patients with antibody deficiency. Allergol Immunopathol (Madrid). 2011;39:128-32.

5. Van de Ven AA, van Montfrans JM, Terheggen-Lagro SW, Beek FJ, Hoytema van Konijnenburg DP, Kessels OA, et al. A CT scan score for the assessment of lung disease in children with common variable immunodeficiency disorders. Chest. 2010;138:371-9.

6. Maarschalk-Ellerbroek LJ, de Jong PA, van Montfrans JM, Lammers JW, Bloem AC, Hoepelman AI, et al. CT screening for pulmonary pathology in common variable immunodeficiency disorders and the correlation with clinical and immunological parameters. J Clin Immunol. 2014;34:642-54.

7. Ballow M. Managing comorbid complications in patients with common variable immunodeficiency. Ann Allergy Asthma Immunol. 2013;111:S6-9. 
8. Prasse A, Kayser G, Warnatz K. Common variable immunodeficiencyassociated granulomatous and interstitial lung disease. Curr Opin Pulm Med. 2013;19:503-9.

9. Park JH, Levinson AI. Granulomatous-lymphocytic interstitial lung disease (GLILD) in common variable immunodeficiency (CVID). Clin Immunol. 2010;134:97-103.

10. Torigian DA, LaRosa DF, Levinson AI, Litzky LA, Miller Jr WT. Granulomatous-lymphocytic interstitial lung disease associated with common variable immunodeficiency: CT findings. J Thorac Imaging. 2008;23:162-9.

11. Bates CA, Ellison MC, Lynch DA, Cool CD, Brown KK, Routes JM. Granulomatous-lymphocytic lung disease shortens survival in common variable immunodeficiency. J Allergy Clin Immunol. 2004;114:415-21.

12. Maglione PJ, Ko HM, Beasley MB, Strauchen JA, CunninghamRundles C. Tertiary lymphoid neogenesis is a component of pulmonary lymphoid hyperplasia in patients with common variable immunodeficiency. J Allergy Clin Immunol. 2014;133:535-42.

13. Da Silva SP, Resnick E, Lucas M, Lortan J, Patel S, CunninghamRundles C, et al. Lymphoid proliferations of indeterminate malignant potential arising in adults with common variable immunodeficiency disorders: unusual case studies and immunohistological review in the light of possible causative events. J Clin Immunol. 2011;31:784-91.

14. Resnick ES, Moshier EL, Godbold JH, Cunningham-Rundles C. Morbidity and mortality in common variable immune deficiency over 4 decades. Blood. 2012;119:1650-7.

15. Quinti I, Agostini C, Tabolli S, Brunetti G, Cinetto F, Pecoraro A, et al. Malignancies are the major cause of death in patients with adult onset common variable immunodeficiency. Blood. 2012;120:1953-4.

16. Offer SM, Pan-Hammarström Q, Hammarström L, Harris RS. Unique DNA repair gene variations and potential associations with the primary antibody deficiency syndromes IgAD and CVID. PLoS ONE. 2010;5:e12260.

17. Vorechovský I, Scott D, Haeney MR, Webster DA. Chromosomal radio sensitivity in common variable immune deficiency. Mutat Res. 1993;290:255-64.

18. Aghamohammadi A, Moin M, Kouhi A, Mohagheghi MA, Shirazi A, Rezaei N, et al. Chromosomal radiosensitivity in patients with common variable immunodeficiency. Immunobiology. 2008;213:447-54.

19. Palanduz S, Palanduz A, Yalcin I, Somer A, Ones U, Ustek D, et al. In vitro chromosomal radio sensitivity in common variable immune deficiency. Clin Immunol Immunopathol. 1998;86:180-2.

20. Serra G, Milito C, Mitrevski M, Granata G, Martini H, Pesce AM, et al. Lung MRI as a possible alternative to CT scan for patients with primary immune deficiencies and increased radiosensitivity. Chest. 2011;140:1581-9.

21. Luna A, Sánchez-Gonzalez J, Caro P. Diffusion-weighted imaging of the chest. Magn Reson Imaging Clin N Am. 2011;19:69-94.

22. Nguyen TL, Soyer P, Barbe C, Graesslin O, Veron S, AmzallagBellenger E, et al. Diagnostic value of diffusion-weighted magnetic resonance imaging in pelvic abscesses. J Comput Assist Tomogr. 2013;37:971-9.

23. Ream JM, Dillman JR, Adler J, Khalatbari S, McHugh JB, Strouse PJ, et al. MRI diffusion-weighted imaging (DWI) in pediatric small bowel Crohn disease: correlation with MRI findings of active bowel wall inflammation. Pediatr Radiol. 2013;43:1077-85.

24. Taouli B, Chouli M, Martin AJ, Qayyum A, Coakley FV, Vilgrain V. Chronic hepatitis: role of diffusion-weighted imaging and diffusion tensor imaging for the diagnosis of liver fibrosis and inflammation. J Magn Reson Imaging. 2008;28:89-95.

25. Tondo F, Saponaro A, Stecco A, Lombardi M, Casadio C, Carriero A. Role of diffusion-weighted imaging in the differential diagnosis of benign and malignant lesions of the chest-mediastinum. Radiol Med. 2011;116:720-33.

26. Satoh S, Kitazume Y, Ohdama S, Kimula Y, Taura S, Endo Y. Can malignant and benign pulmonary nodules be differentiated with diffusion-weighted MRI? AJR Am J Roentgenol. 2008;191:464 70.

27. Liu H, Liu Y, Tielian Y, Ye N. Usefulness of diffusion-weighted MR imaging in the evaluation of pulmonary lesions. Eur Radiol. 2010;20:807-15.

28. Conley ME, Notarangelo LD, Etzioni A. Diagnostic criteria for primary immunodeficiencies: representing PAGID (Pan-American Group for Immunodeficiency) and ESID (European Society for Immunodeficiencies). Clin Immunol. 1999;93:190-7.

29. Bhalla M, Turcios N, Aponte V, Jenkins M, Leitman BS, McCauley DI, et al. Cystic fibrosis: scoring system with thin-section CT. Radiology. 1991;179:783-8.

30. Padley SP, Adler B, Muller NL. High-resolution computed tomography of the chest: current indications. J Thorac Imaging. 1993;8: 189-99.

31. Montella S, Mollica C, Finocchi A, Pession A, Pietrogrande MC, Trizzino A, et al. Non invasive assessment of lung disease in ataxia telangiectasia by high-field magnetic resonance imaging. J Clin Immunol. 2013;33:1185-91.

32. Lutterbey G, Grohé C, Gieseke J, von Falkenhausen M, Morakkabati N, Wattjes MP, et al. Initial experience with lungMRI at 3.0 T: comparison with CT and clinical data in the evaluation of interstitial lung disease activity. Eur J Radiol. 2007;61:25661.

33. Sheth VR, van Heeckeren RC, Wilson AG, van Heeckeren AM, Pagel MD. Monitoring infection and inflammation in murine models of cystic fibrosis with magnetic resonance imaging. J Magn Reson Imaging. 2008;28:527-32.

34. Klein M, Cohen-Cymberknoh M, Armoni S, Shoseyov D, Chisin $\mathrm{R}$, Orevi M, et al. F-fluorodeoxyglucose-PET/CT imaging of lungs in patients with cystic fibrosis. Chest. 2009;136:1220-8.

35. Amin R, Charron M, Grinblat L, Shammas A, Grasemann H, Graniel K, et al. Cystic fibrosis: detecting changes in airway inflammation with FDG PET/CT. Radiology. 2012;264:868-75.

36. Uto T, Takehara Y, Nakamura Y, Naito T, Hashimoto D, Inui N, et al. Higher sensitivity and specificity for diffusion-weighted imaging of malignant lung lesions without apparent diffusion coefficient quantification. Radiology. 2009;252:247-54.

37. Kuruvilla M, de la Morena MT. Antibiotic prophylaxis in primary immune deficiency disorders. J Allergy Clin Immunol Pract. 2013;1:573-82.

38. Burtin $\mathrm{C}$, Hebestreit $\mathrm{H}$. Rehabilitation in patients with chronic respiratory disease other than chronic obstructive pulmonary disease: Exercise and physical activity interventions in cystic fibrosis and non-cystic Fibrosis Bronchiectasis. Respiration, 2015 Feb 12.

39. Rakhmanov M, Gutenberger S, Keller B, Schlesier M, Peter HH, Warnatz K. CD21low B cells in common variable immunodeficiency do not show defects in receptor editing, but resemble tissue-like memory B cells. Blood. 2010;116:3682-3.

40. Park J, Munagala I, Xu H, Blankenship D, Maffucci P, Chaussabel $\mathrm{D}$, et al. Interferon signature in the blood in inflammatory common variable immune deficiency. PLoS ONE. 2013;8:e74893.

41. Salzer U, Warnatz K, Peter HH. Common variable immunodeficiency - an update. Arthritis Res Ther. 2012;14:223.

42. Chase NM, Verbsky JW, Hintermeyer MK, Waukau JK, TomitaMitchell A, Casper JT, et al. Use of combination chemotherapy for treatment of granulomatous and lymphocytic interstitial lung disease (GLILD) in patients with common variable immunodeficiency (CVID). 2013,33:30-9.

43. Franxman TJ, Howe LE, Baker JR Jr J. Infliximab for treatment of granulomatous disease in patients with common variable immunodeficiency. Clin Immunol. 2014;34:820-7.

44. Cunningham-Rundles C. Lung disease, antibodies and other unresolved issues in immune globulin therapy for antibody deficiency. Clin Exp Immunol. 2009;157:12-6. 